\title{
Association of Preoperative Serum Carcinoembryonic Antigen and Gastric Cancer Recurrence: A Large Cohort Study
}

\author{
Qinbo Cai ${ }^{1,2,3^{*}}$, Wen Zhou ${ }^{3 *}$, Jin $\mathrm{Li}^{1,2,3,4}$, Xinde Ou${ }^{1,2,3}$, Chuangqi Chen ${ }^{1,2}$, Shirong Cai ${ }^{1,2}$, Weiling $\mathrm{He}^{1,2}{ }^{\bowtie}$, \\ Jianbo $\mathrm{Xu}^{1,2}$ and Yulong $\mathrm{He}^{1,2,4}$ \\ 1. Center of Gastrointestinal Surgery, the First Affiliated Hospital of Sun Yat-sen University, Guangzhou, P. R. China. \\ 2. Center for Diagnosis and Treatment of Gastric Cancer, Sun Yat-sen University, Guangzhou, P. R. China. \\ 3. Laboratory of General Surgery, the First Affiliated Hospital of Sun Yat-sen University, Guangzhou, P. R. China. \\ 4. Center for Digestive Disease, the Seventh Affiliated Hospital of Sun Yat-sen University, Shenzhen, P. R. China. \\ *These authors contributed equally to this study. \\ $\triangle$ Corresponding authors: Yulong He, MD, PhD, Tel.: +86 2087755766 8211, Fax: +86 20 28823389, E-mail: heyulong@mail.sysu.edu.cn; Jianbo Xu, MD, PhD, \\ E-mail: xjianb@mail.sysu.edu.cn; or Weiling He, MD, PhD, E-mail: hewling@mail.sysu.edu.cn. Tel.: +86 20 877557668211 , Fax: +86 20 28823389.
}

(C) The author(s). This is an open access article distributed under the terms of the Creative Commons Attribution License (https://creativecommons.org/licenses/by/4.0/). See http://ivyspring.com/terms for full terms and conditions.

Received: 2020.05.07; Accepted: 2020.10.25; Published: 2021.01.01

\begin{abstract}
Background and Aim: Measuring postoperative carcinoembryonic antigen (CEA) is recommended by guidelines to help detecting recurrence of gastric cancer patients. However, the prognostic significance of elevated preoperative CEA is unclear. This study aims to investigate whether patients with elevated preoperative CEA have a higher risk of recurrence than patients with normal preoperative CEA.

Methods: We conducted a retrospective cohort study at a gastric cancer center in South China. Consecutive patients with stage I to III gastric adenocarcinoma who underwent curative resection at the center from January 2001 to February 2016 were identified. Patients were grouped into two cohorts: normal preoperative CEA ( $\leq 5 \mathrm{ng} / \mathrm{ml}$ ), and elevated preoperative CEA (> $5 \mathrm{ng} / \mathrm{ml}$ ). 3-year recurrence-free survival (RFS) and hazard function curves over time were estimated.

Results: A total of 1,596 patients $(1,063\{66.6 \%\}$ male; median $\{$ Interquartile range, IQR $\}$ age, $59\{50-66\}$ years) were identified. Patients with elevated preoperative CEA had 15.5\% lower 3-year RFS $(n=222$ $\{70.4 \%\})$ than the cohorts with normal preoperative CEA $(n=1,374\{85.9 \%\})$. The hazard function of recurrence for the two cohorts peaked at the similar time (around 10 months after surgery). Multivariate Cox analyses confirmed that elevated preoperative CEA was independently associated with shorter RFS (Hazard Ratio $\{H R\}, 1.69 ; 95 \%$ confidence interval $\{\mathrm{Cl}\}, 1.26-2.27 ; P=0.001$ ).

Conclusions: Patients with elevated preoperative CEA are at increased risk for recurrence, especially within the first 24 months after surgery.
\end{abstract}

Key words: Gastric cancer; CEA; recurrence; prognostic factor

\section{Introduction}

Carcinoembryonic antigen (CEA) is recommended by national guidelines as a tumor marker in gastric cancer [1, 2]. Previous studies had controversial results about association between preoperative serum CEA and overall survival of gastric cancer even in large sample size cohorts [3-7]. Due to lack of high quality evidence, level of preoperative CEA is not considered as independent prognostic factor for gastric cancer, and cure strategy should not be changed based on preoperative CEA level according to the $8^{\text {th }}$ edition of American Joint Committee (AJCC) staging system [8]. Overall survival is affected by various non-cancer factors, such as non-gastric cancer diseases and income, which are hard to be adjusted in multivariate analysis and might lead to bias. Recurrence heralds a worse 
prognosis after radical resection of gastric cancer [9] and is supposed to be an indicator for cancer-specific outcome. Measuring postoperative CEA has been recommended by guidelines to help detecting recurrence $[1,2,8,10]$, but the role of preoperative CEA in predicting recurrence is still unclear. There is not cohort with large size of patients reporting the association between preoperative CEA and recurrence of gastric cancer. In this study, we aimed to determine whether preoperative serum CEA is a prognostic factor for recurrence of gastric cancer after radical resection in a large sample size cohort.

\section{Materials and Methods}

\section{Study design and patient cohort}

This study was approved by the institutional review board of the First Affiliated Hospital of Sun Yat-sen University. Informed consent of study patients was waived by the review board. Inclusion criteria: Prospectively maintained databases were queried for all consecutive patients who underwent curative surgery for stage I to III gastric cancer patients from January 2001 to February 2016 at gastric cancer center of the First Affiliated Hospital of Sun Yat-sen University. The last follow-up date of this study was February 2018. Exclusion criteria: Preoperative chemotherapy or radiotherapy, lack of preoperative CEA data, non-curative palliative resection, gastric stump carcinoma, death of surgery complication, presence of other malignant tumors, non-available $\mathrm{T}$ stage or $\mathrm{N}$ stage, and nonadenocarcinoma (Figure 1).

Preoperative serum CEA was defined as the CEA value closest to surgical date. Patients were grouped as follows: (1) Normal preoperative CEA group ( $\leq 5 \mathrm{ng} / \mathrm{ml}$ ); (2) Elevated preoperative CEA group (> $5 \mathrm{ng} / \mathrm{ml}$ ). The CEA value was measured at the First Affiliated Hospital of Sun Yat-sen University using an Abott ARCHITECT analyzer (Abott, USA). The reference normal range was 0.0 to $5.0 \mathrm{ng} / \mathrm{ml}$.

\section{Staging and follow-up}

The preoperative stage was determined by contrast-enhanced computed tomography (CT) of the chest, abdomen and pelvis. The original pathologic TNM stages of patients in the databases were based on the $6^{\text {th }}$ and $7^{\text {th }}$ AJCC on Cancer staging system. The original pathological reports were reviewed by clinician, and then modified all the pathologic stages according to the $8^{\text {th }}$ edition [8]. Adjuvant chemotherapy was administered to patients after histological evaluation of the surgical specimen according to the National Comprehensive Cancer Network (NCCN) guidelines. Postoperative followup was performed every 3-6 months for the first 3 years, then every 12 months from years 4 to 5 . The routine patient follow-up appointments included a

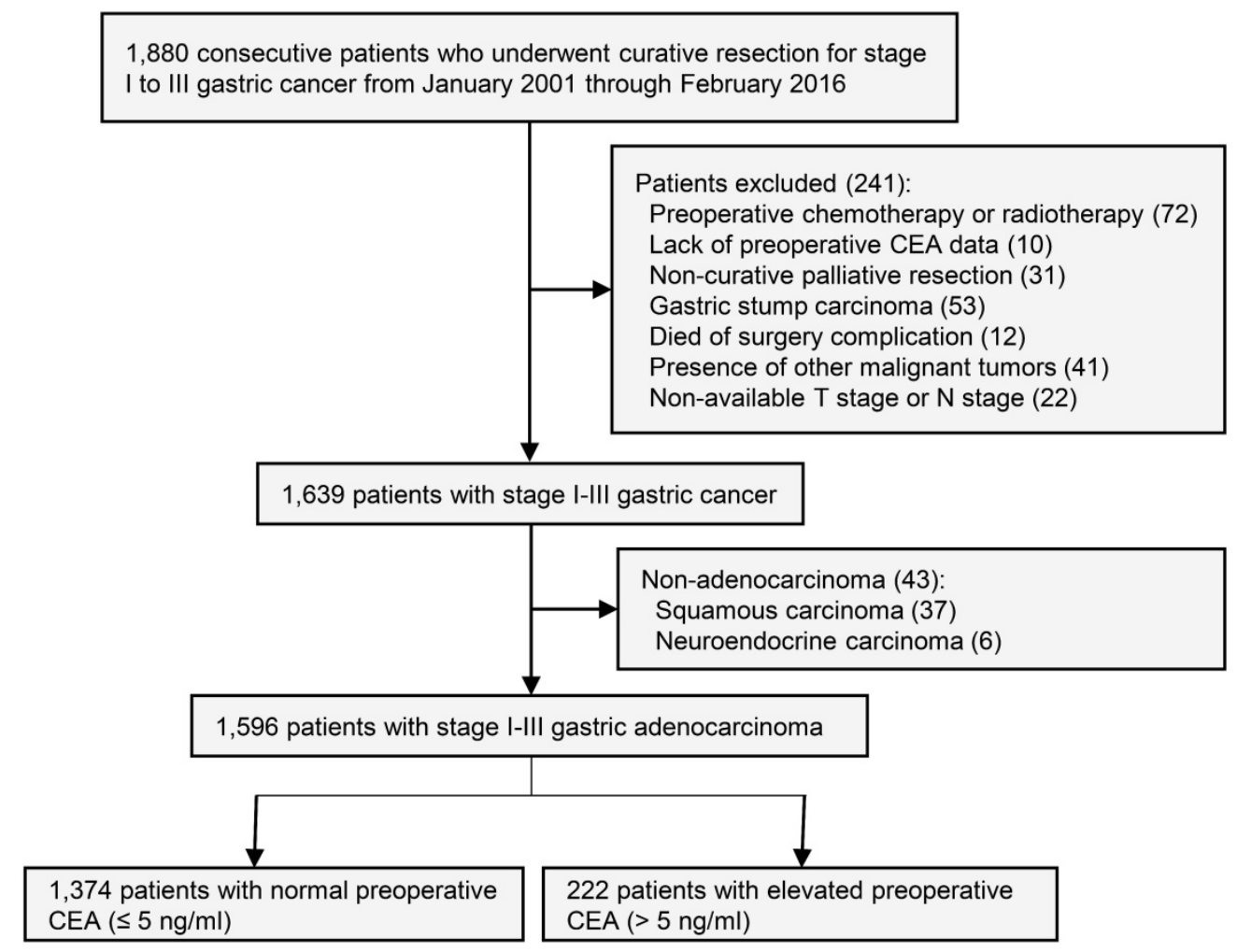

Figure 1. Study design. 
physical examination, laboratory tests, chest radiography, abdominal ultrasonography, CT or positron emission computed tomography (PETCT) and an annual endoscopic examination. CT was the most frequently used imaging method, including chest, abdomen and pelvis with intravenous contrast. Diagnosis of recurrence was based on new lesions on CT, PETCT or histological confirmation through biopsy.

\section{Statistical analysis}

Continuous variables were compared using the Mann-Whitney $U$ test. Categorical variables were compared using the Chi-square test or Fisher's exact test. The recurrence-free survival (RFS) period was defined as the period from the date of surgery to the date of recurrence or last follow-up without recurrence. Patients who died without known tumor recurrence were censored at the last documented evaluation [11]. Differences in RFS were assessed by the Log-rank test. Hazard ratio (HR) and 95\% confidence interval $(\mathrm{CI})$ were estimated using Cox regression models and evaluated by the Wald test. Variables with $P$ value less than 0.05 on univariate analyses were included in the multivariate analysis. The hazard function of recurrence was estimated using Kernel-based method [12, 13]. All statistical analyses were performed using $\mathrm{R}$ version 3.6.1 ( $\mathrm{R}$ project) and IBM SPSS software (version 22, New York, USA). All tests were two-sided and $P$ values of less than 0.05 were considered significant.

\section{The Cancer Genome Atlas (TCGA) stomach adenocarcinoma analysis}

We downloaded mRNA expression data and clinical information of the TCGA stomach adenocarcinoma program from cBioPortal. Patients with stage I-III gastric adenocarcinoma were included. Exclusion criteria as follows: Patients with preoperative chemotherapy or radiotherapy, or with other malignant tumor. Patients with CEA cell adhesion molecule 5 (CEACAM5, name of gene encoding protein CEA) expression in the top $20 \%$ range were divided into high expression group, and the rest $80 \%$ were divided into low expression group.

\section{In silico mechanism analysis}

We used cBioPortal to identify genes correlated with CEACAM5 in mRNA expression level. Genes with an adjusted- $P$ value less than 0.01 were considered significantly correlated with CEACAM5 and used for Gene Oncology (GO) enrichment and Kyoto Encyclopedia of Genes and Genomes (KEGG) pathways analyses. The clusterProfiler $R$ package was used for analysis.

\section{Results}

A total of 1,596 patients $(1,063\{66.6 \%\}$ male; median $\{$ Interquartile range, IQR $\}$ age, $59\{50-66\}$ years) were identified. Characteristics of the 1,596 patients with normal or elevated preoperative CEA were shown in Table 1. In this study, 1,374 patients were grouped into the normal preoperative CEA cohort with a median (IQR) CEA level of 1.6 (0.99-2.49) $\mathrm{ng} / \mathrm{ml}$. The elevated preoperative CEA cohort included 222 patients and the median (IQR) CEA level was 11.09 (7.04-27.18). The median (IQR) follow-up time for all patients was 37.68 (18.17-60.00) months. A total of 253 patients $(15.9 \%)$ had recurrence before the last follow-up. The 3-year RFS rate for all patients was $83.8 \%$ (95\% CI, $81.8 \%-85.9 \%$ ).

Table 1. Patients and tumor characteristics

\begin{tabular}{|c|c|c|c|}
\hline Characteristics & $\begin{array}{l}\mathrm{CEA} \leq 5 \mathrm{ng} / \mathrm{ml} \\
(\mathrm{n}=1,374)\end{array}$ & $\begin{array}{l}\text { CEA > } 5 \mathrm{ng} / \mathrm{ml} \\
(\mathrm{n}=222)\end{array}$ & $P$ \\
\hline \multicolumn{3}{|l|}{ Gender, n (\%) } & $<0.001$ \\
\hline Male & $889(64.7)$ & $174(78.4)$ & \\
\hline Female & $485(35.3)$ & $48(21.6)$ & \\
\hline Age, years, median (IQR) & $58(49-66)$ & $63(55-69)$ & $<0.001$ \\
\hline \multicolumn{3}{|l|}{ Primary site, n (\%) } & $<0.001$ \\
\hline Upper third & $354(25.8)$ & $93(41.9)$ & \\
\hline Middle third & $358(26.1)$ & $51(23.0)$ & \\
\hline Lower third & $620(45.1)$ & $72(32.4)$ & \\
\hline Entire & $41(3.0)$ & $6(2.7)$ & \\
\hline Unknown & $1(0.1)$ & $0(0)$ & \\
\hline \multicolumn{3}{|c|}{ Tumor differentiation, $\mathbf{n}(\%)$} & 0.862 \\
\hline $\mathrm{G} 1 / 2$ & $355(25.8)$ & $61(27.5)$ & \\
\hline G3/4 & $990(72.1)$ & $156(70.3)$ & \\
\hline Gx & $29(2.1)$ & $5(2.3)$ & \\
\hline $\begin{array}{l}\text { No. of retrieved lymph } \\
\text { nodes, Median (IQR) }\end{array}$ & $29(12-41)$ & $32.5(21-42.25)$ & 0.036 \\
\hline \multicolumn{3}{|l|}{ Pathologic T stage, $n(\%)$} & $<0.001$ \\
\hline Tis/T1 & $280(20.4)$ & $14(6.3)$ & \\
\hline $\mathrm{T} 2$ & $146(10.6)$ & $16(7.2)$ & \\
\hline T3 & $188(13.7)$ & $32(14.4)$ & \\
\hline T4a & $666(48.5)$ & $136(61.3)$ & \\
\hline $\mathrm{T} 4 \mathrm{~b}$ & $94(6.8)$ & $24(10.8)$ & \\
\hline \multicolumn{3}{|l|}{ Pathologic N stage, n (\%) } & $<0.001$ \\
\hline No & $601(43.7)$ & $47(21.2)$ & \\
\hline N1 & $244(17.8)$ & $43(19.4)$ & \\
\hline $\mathrm{N} 2$ & $258(18.8)$ & $50(22.5)$ & \\
\hline N3a & $180(13.1)$ & $51(23.0)$ & \\
\hline $\mathrm{N} 3 \mathrm{~b}$ & $91(6.6)$ & $31(14.0)$ & \\
\hline \multicolumn{3}{|c|}{ AJCC $8^{\text {th }}$ ed. pathologic stage, $n(\%)$} & $<0.001$ \\
\hline $\mathrm{I}^{*}$ & $359(26.1)$ & $19(8.6)$ & \\
\hline II & $340(24.7)$ & $41(18.5)$ & \\
\hline III & $675(49.1)$ & $162(73.0)$ & \\
\hline \multicolumn{3}{|c|}{ Adjuvant chemotherapy, $\mathrm{n}(\%)$} & 0.170 \\
\hline Yes & $816(59.4)$ & $121(54.5)$ & \\
\hline No & $558(40.6)$ & $101(45.5)$ & \\
\hline
\end{tabular}

*There were 19 patients with stage 0 disease and they were grouped into stage I during analyses.

In the normal group and the elevated group, the median (IQR) follow-up periods were 39.92 (18.9-60) and 25.69 (10.69-58.02) months, respectively. The 1-year RFS rate for patients with elevated preoperative CEA was $86.1 \%$ (95\% CI, $81.4 \%-91.0 \%$ ) 
compared with $94.8 \%$ (95\% CI, 93.6\%-96.0\%) for patients with normal preoperative CEA. The 3-year RFS rate for elevated group was $70.4 \%(95 \% \mathrm{CI}$, $63.9 \%-77.5 \%)$ compared with $85.9 \% \quad(95 \% \quad \mathrm{CI}$, $83.9 \%-88.0 \%$ ) for normal group (Figure 2A).

The smooth curve of the hazard function indicated that the risk of recurrence was higher in the elevated preoperative CEA group (Figure 2B). Nevertheless, both of the two groups peaked at the similar time and had high recurrence risk during the first 2 years after surgery.

We further investigated relationship between RFS and CEA in different stage patients (Figure 2C). The RFS of two cohorts had no significant difference in patients with stage I or II gastric cancer (Figure 2C). However, among patients with stage III gastric cancer, RFS was significantly lower in the elevated preoperative CEA cohort than that of normal preoperative CEA cohort (3-year RFS rate, $60.9 \%$ vs 74.8\%, Log-rank $P=3.5 \mathrm{E}-04$ ) (Figure 2C).

Table 2. Univariate and multivariate analyses of recurrence-free survival

\begin{tabular}{|c|c|c|c|c|c|c|}
\hline \multirow[t]{2}{*}{ Characteristics } & \multicolumn{3}{|c|}{ Univariate analysis } & \multicolumn{3}{|c|}{ Multivariate analysis } \\
\hline & HR & $95 \% \mathrm{CI}$ & $P$ & HR & $95 \% \mathrm{CI}$ & $P$ \\
\hline \multicolumn{7}{|l|}{ Gender } \\
\hline Male & Ref & & & & & \\
\hline Female & 1.17 & $0.90-1.51$ & 0.236 & & & \\
\hline \multicolumn{7}{|l|}{ Age, years } \\
\hline$\leq 55$ & Ref & & & & & \\
\hline $55-60$ & 0.79 & $0.53-1.16$ & 0.226 & & & \\
\hline $60-65$ & 1.05 & $0.73-1.50$ & 0.788 & & & \\
\hline$>65$ & 1.14 & $0.85-1.54$ & 0.387 & & & \\
\hline \multicolumn{7}{|l|}{ Primary site } \\
\hline Upper third & 0.50 & $0.28-0.89$ & 0.019 & 0.69 & $0.38-1.25$ & 0.224 \\
\hline Middle third & 0.42 & $0.24-0.77$ & 0.005 & 0.68 & $0.38-1.24$ & 0.208 \\
\hline Lower third & 0.30 & $0.17-0.54$ & $<0.001$ & 0.54 & $0.30-0.97$ & 0.039 \\
\hline Entire & Ref & & & Ref & & \\
\hline \multicolumn{7}{|c|}{ Tumor differentiation } \\
\hline $\mathrm{G} 1 / 2$ & Ref & & & Ref & & \\
\hline $\mathrm{G} 3 / 4$ & 2.08 & $1.49-2.90$ & $<0.001$ & 1.65 & $1.17-2.33$ & 0.004 \\
\hline \multicolumn{7}{|c|}{$\begin{array}{l}\text { No. of retrieved lymph } \\
\text { nodes }\end{array}$} \\
\hline$<16$ & Ref & & & & & \\
\hline$\geq 16$ & 1.24 & $0.88-1.75$ & 0.224 & & & \\
\hline \multicolumn{7}{|c|}{ AJCC $8^{\text {th }}$ ed. pathologic stage } \\
\hline I & Ref & & & Ref & & \\
\hline II & 5.63 & 2.64-11.98 & $<0.001$ & 5.21 & $2.32-11.69$ & $<0.001$ \\
\hline III & 17.13 & $8.45-34.73$ & $<0.001$ & 14.71 & $6.83-31.67$ & $<0.001$ \\
\hline \multicolumn{7}{|c|}{ Adjuvant chemotherapy } \\
\hline Yes & Ref & & & Ref & & \\
\hline No & 1.67 & $1.30-2.14$ & $<0.001$ & 1.01 & $0.79-1.31$ & 0.923 \\
\hline \multicolumn{7}{|l|}{ CEA } \\
\hline$\leq 5 \mathrm{ng} / \mathrm{ml}$ & Ref & & & Ref & & \\
\hline$>5 \mathrm{ng} / \mathrm{ml}$ & 2.31 & $1.73-3.09$ & $<0.001$ & 1.69 & $1.26-2.27$ & 0.001 \\
\hline
\end{tabular}

Univariate and multivariate analyses for risk factors associated with RFS are shown in Table 2. In univariate analyses, tumor located in entire stomach, poor differentiation, higher TNM stage, no adjuvant chemotherapy and elevated preoperative CEA were associated with shorter RFS. Multivariate analysis indicated that elevated preoperative CEA was independently associated with shorter RFS (HR = $1.69,95 \% \mathrm{CI}=1.26-2.27, P=0.001)$ together with tumor located in entire stomach, poor differentiation and higher TNM stage.

The recurrence patterns of the two cohorts were shown in Table 3. It was interesting that patients with elevated preoperative CEA was more likely to have liver metastasis rather than peritoneal metastasis.

Table 3. Analysis of sites of recurrence in relation to CEA status

\begin{tabular}{|c|c|c|c|}
\hline Recurrence site & $\mathrm{CEA} \leq 5 \mathrm{ng} / \mathrm{ml}, \mathrm{n}(\%)$ & CEA > $5 \mathrm{ng} / \mathrm{ml}, \mathrm{n}(\%)$ & $P^{\#}$ \\
\hline Total sites & 185 & 55 & \\
\hline Local & & & 0.059 \\
\hline Present & $25(13.51)$ & $14(25.45)$ & \\
\hline Absent & $160(86.49)$ & $41(74.55)$ & \\
\hline Peritoneal & & & 0.003 \\
\hline Present & $78(42.16)$ & $11(20.00)$ & \\
\hline Absent & $107(57.84)$ & $44(80.00)$ & \\
\hline Lymph node & & & 0.372 \\
\hline Present & $28(15.14)$ & $5(9.09)$ & \\
\hline Absent & $157(84.86)$ & $50(90.91)$ & \\
\hline Liver & & & 0.011 \\
\hline Present & $29(15.68)$ & $18(32.73)$ & \\
\hline Absent & $156(84.32)$ & $37(67.27)$ & \\
\hline Lung & & & 0.822 \\
\hline Present & $24(12.97)$ & $8(14.55)$ & \\
\hline Absent & $161(87.03)$ & $47(85.45)$ & \\
\hline Other sites* & & & 0.414 \\
\hline Present & $14(7.57)$ & $6(10.91)$ & \\
\hline Absent & $171(92.43)$ & $49(89.09)$ & \\
\hline
\end{tabular}

To validate whether the mRNA level of CEA was associated with recurrence of gastric cancer, we downloaded mRNA expression data of CEACAM5 and clinical information from cBioPortal. Results showed that high expression of CEACAM5 was significantly associated with poor RFS. The 3-year RFS rate for high-expression group was 48.3\% (95\% CI, $34.4 \%-68.0 \%)$ compared with $58.1 \% \quad(95 \% \quad \mathrm{CI}$, $50.5 \%-66.8 \%$ ) for low-expression group (Figure S1A). The same trend was observed in the subgroup analyses of each stage. However, there was no significant statistical difference, which might be due to the small sample size in each stage (Figure S1 B-D).

In order to investigate the possible mechanisms associated with CEA and gastric cancer recurrence, cBioPortal was used to analyze genes correlated with CEACAM5 in mRNA expression level. Finally, 3,286 genes with an adjusted- $P$ value less than 0.01 were considered significantly correlated with CEACAM5 and included for GO enrichment and KEGG signaling pathways analyses (Table S1). As a membrane protein, CEA was associated with cell-cell adhesion and junction (Figure S2) [14]. Surprisingly, as indicated by results from both GO and KEGG, CEA 
was significantly associated with chemokine signaling and immunology regulation, especially $\mathrm{T}$ cells and $\mathrm{Th}$ cells (Figure S2), which were already known to mediate cancer metastasis in previous studies [15-17].

\section{Discussion}

In this study, we observed that patients with elevated preoperative CEA have a 15.5\% lower 3-year RFS than those with normal preoperative CEA. The hazard function curve further demonstrated the impact of elevated CEA and shown a higher peak in the elevated CEA cohort compared with the normal CEA cohort. Multivariate Cox regression also confirmed that elevated CEA was an independent prognostic factor for recurrence of gastric cancer. In stratified analysis, we observed that preoperative CEA could stratify patients with stage III rather than those with stage I or II, though patients with elevated CEA have a $10.4 \%$ lower 1-year RFS than those with normal CEA in stage II without statistical significance. This difference is likely due to the limited recurrence in stage I group, which has a 3-year RFS greater than 98\%.

Preoperative CEA was reported as an independent prognostic factor for RFS in a retrospective cohort of 621 patients by multivariate Cox model, adjusted by for age, stage, NUAK family kinase 2 (NUAK2), pyruvate dehydrogenase kinase 1 (PDK1), phospho-AMP-activated protein kinase (pAMPK) and mitogen activated kinase (MAPK) 3/1 [18]. Other studies also reported elevated preoperative CEA predicted shorter RFS under unadjusted condition [19-21], while some studies showed negative results without adjustment [22, 23], and the sample size of these studies vary from 70 to 479. The results from these studies are inconsistent, which might be due to the small sample size and unadjusted analyses. In this study, we demonstrated the prognostic value of preoperative CEA using multivariate analysis in a large cohort of 1,596 consecutive patients. We intend to provide some evidence for clinic practice.
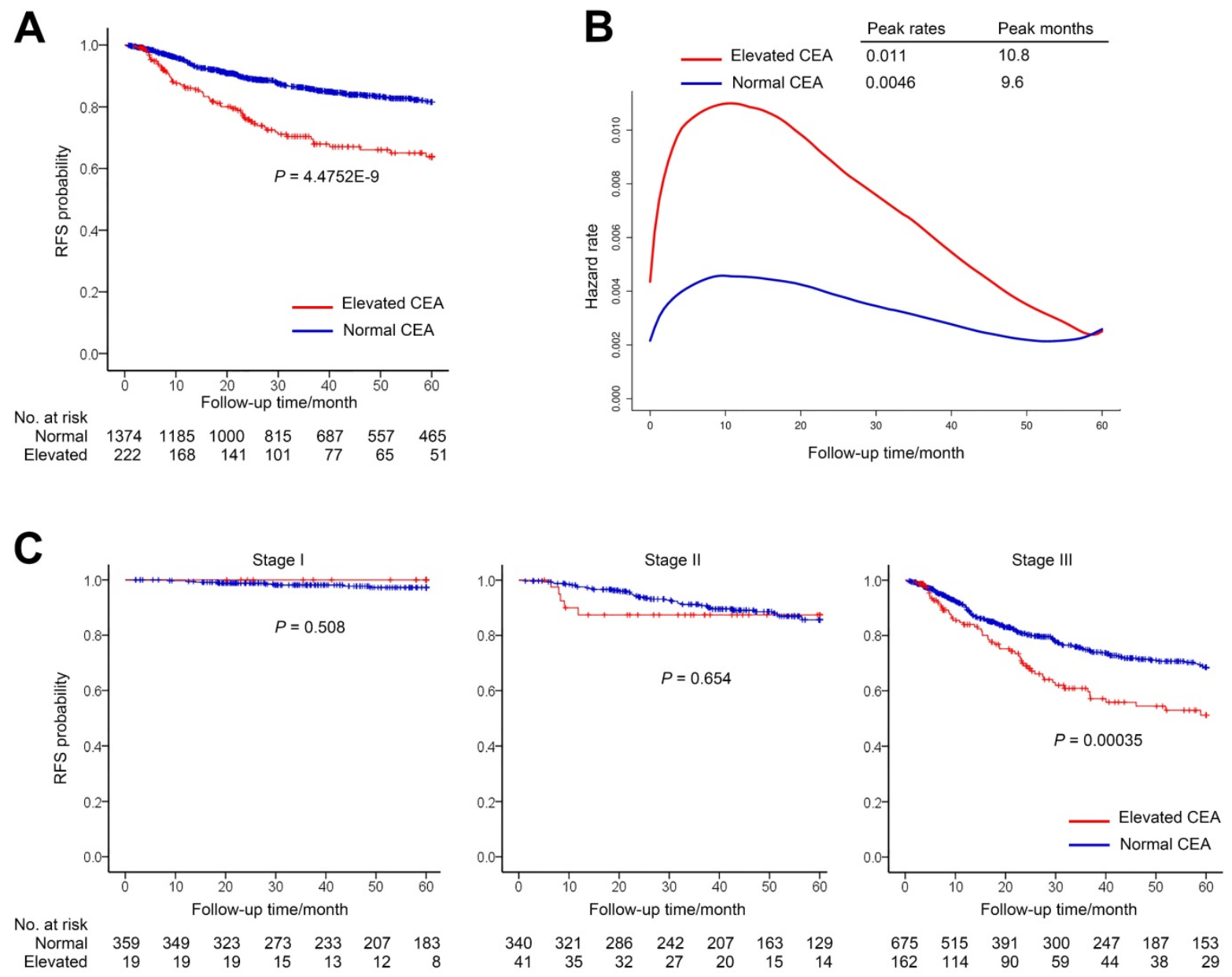

Figure 2. Recurrence-free survival (RFS) by preoperative CEA. (A) RFS of patients with normal preoperative CEA vs elevated preoperative CEA. (B) Hazard functions for the disease recurrence in the two cohorts. (C) Stage-specific analyses of RFS based on preoperative CEA level. 
The important role of postoperative CEA in cancer surveillance has been widely accepted $[1,2,8$, 10]. Since some elevated preoperative CEA normalized after surgery [5], people may argue that preoperative CEA is not needed for follow-up. However, recurrence can be accompanied by normal or elevated postoperative CEA [22]. The value of elevated preoperative CEA is to define a small proportion of patients $(13.9 \%$ in this study, $16.6 \%$ to $28.8 \%$ in other studies $[3,5,6])$ with higher risk of recurrence compared to patients with normal preoperative CEA in the same stage. These patients are supposed to undergo more intensive surveillance plan. Besides serum CEA, positive of preoperative CEA mRNA in peritoneal lavages predicts the peritoneal recurrence [24], which confirms the role of CEA in defining patients with high risk of recurrence.

This study indicates that patients with elevated CEA were more likely to have liver metastasis than those with normal CEA as previously reported [25, 26]. Higher CEA is also associated with positive vessel carcinoma embolus [27]. It seems that tumors with elevated CEA are more invasive and prefer hematogenous metastasis. CEA is shown to inhibit transforming growth factor- $\beta$ (TGF- $\beta$ ) signaling [28] and promotes liver metastasis of colon cancer in mice model [28, 29].

The major strengths of this study include large size of the cohort, patients with high-quality preoperative $\mathrm{CT}$ and standardized pathological reports; the uniform treatment patients received, with standard D2 or D2+ resection technique by specialized gastric surgeons and retrieved lymph node more than 16 in $83.2 \%$ of patients in this cohort; and standard adjuvant chemotherapy in all patients. Standard chemotherapy was handled by a special group in our center over years. This cohort has similar oncological outcomes to those seen in other reports from centers in Japan and Korea, where standard D2 resection technique is commonly performed. For example, the recurrence rate in this cohort is similar as previous report [30].

\section{Limitations}

This study is with limitation inherent in observational retrospective cohorts. First, intervals and completeness of the follow-up were varied, although postoperative follow-up was performed according to national guideline [1]. Second, patients who died without known tumor recurrence were censored in this study. It is possible that some of them died because of recurrence without imaging test, which leads to underestimation of recurrence rate and potential bias. In addition, we did not control known factors that affect CEA, such as tobacco use, nonmalignant gastrointestinal disorders, lung disease, and hypothyroidism [31, 32]. Furthermore, this study is implemented in a single center and it would have some limitations to apply to other centers. Further validation studies are warranted to explore the value of preoperative CEA as an independent prognostic factor.

\section{Conclusion}

The elevated preoperative CEA independently predicts shorter RFS for patients with radically resected gastric cancer. Patients with elevated CEA tend to be under significant higher risk of recurrence in the first 2 years after surgery, which might provide evidence for risk-adjusted individualized surveillance strategy.

\section{Abbreviations}

AJCC: American Joint Committee; CEA: carcinoembryonic antigen; CEACAM5: CEA cell adhesion molecule 5; CI: confidence interval; CT: computed tomography; GO: gene oncology; IQR: interquartile range; KEGG: Kyoto Encyclopedia of Genes and Genomes; MAPK: mitogen activated kinase; NCCN: National Comprehensive Cancer Network; NUAK2: NUAK family kinase 2; pAMPK: phospho-AMP-activated protein kinase; PETCT: positron emission computed tomography; PDK1: pyruvate dehydrogenase kinase 1; RFS: recurrencefree survival; TCGA: The Cancer Genome Atlas; TGF- $\beta$ : transforming growth factor- $\beta$.

\section{Supplementary Material}

Supplementary figures.

http://www.jcancer.org/v12p0397s1.pdf

Supplementary table S1.

http://www.jcancer.org/v12p0397s2.xlsx

\section{Acknowledgements}

This study was supported by the National Natural Foundation of China (81472260, 81772579, 81672343 and 81871915), Natural Science Foundation of Guangdong, China (2015A030313053, 2016 A020213002 and 2017A030313570). Science and Technology Program of Guangzhou, China (201510010146 and 201607010050). The Fundamental Research Funds for the Central Universities (15ykpy17) and "3 and 3" Project of The First Affiliated Hospital of Sun Yat-sen University (Yulong He). We appreciate help from all the staffs in the Department of Gastrointestinal Surgery, First Affiliated Hospital of Sun Yat-sen University.

\section{Author Contributions}

YLH: Designed and directed the studies; QBC 
and WZ: Collected data, performed analyses and wrote the manuscript; JL and XDO: Performed the in silico mechanism analyses; CQC and SRC: Revised the manuscript for important intellectual content. WLH and JBX: Directed the in silico mechanism analyses, reviewed and edited the manuscript.

\section{Competing Interests}

The authors have declared that no competing interest exists.

\section{References}

1. Wang FH, Shen L, Li J, Zhou ZW, Liang H, Zhang XT, et al. The Chinese Society of Clinical Oncology (CSCO): clinical guidelines for the diagnosis and treatment of gastric cancer. Cancer communications (London, England). 2019; 39: 10 .

2. National Comprehensive Cancer Network (NCCN). Clinical Practice Guidelines in Oncology: Gastric Cancer. Version 1.2020. FortWashington, PA: NCCN. 2020.

3. Nakane Y, Okamura S, Akehira K, Boku T, Okusa T, Tanaka K, et al. Correlation of preoperative carcinoembryonic antigen levels and prognosis of gastric cancer patients. Cancer. 1994; 73: 2703-8.

4. Jiang X, Hiki N, Nunobe S, Kumagai K, Kubota T, Aikou S, et al. Prognostic importance of the inflammation-based Glasgow prognostic score in patients with gastric cancer. British journal of cancer. 2012; 107: 275-9.

5. Kochi M, Fujii M, Kanamori N, Kaiga T, Kawakami T, Aizaki K, et al. Evaluation of serum CEA and CA19-9 levels as prognostic factors in patients with gastric cancer. Gastric cancer : official journal of the International Gastric Cancer Association and the Japanese Gastric Cancer Association. 2000; 3: $177-86$

6. Kodera Y, Yamamura Y, Torii A, Uesaka K, Hirai T, Yasui K, et al. The prognostic value of preoperative serum levels of CEA and CA19-9 in patients with gastric cancer. The American journal of gastroenterology. 1996; 91: 49-53.

7. Lin JX, Wang W, Lin JP, Xie JW, Wang JB, Lu J, et al. Preoperative Tumor Markers Independently Predict Survival in Stage III Gastric Cancer Patients: Should We Include Tumor Markers in AJCC Staging? Annals of surgical oncology. 2018; 25: 2703-12

8. Amin MB ES, Greene FL, Brierley JD. AJCC cancer staging manual. 8. New York: Springer. 2017.

9. D'Angelica M, Gonen M, Brennan MF, Turnbull AD, Bains M, Karpeh MS. Patterns of initial recurrence in completely resected gastric adenocarcinoma. Annals of surgery. 2004; 240: 808-16.

10. Zaanan A, Bouche O, Benhaim L, Buecher B, Chapelle N, Dubreuil O, et al. Gastric cancer: French intergroup clinical practice guidelines for diagnosis, treatments and follow-up (SNFGE, FFCD, GERCOR, UNICANCER, SFCD, SFED, SFRO). Digestive and liver disease: official journal of the Italian Society of Gastroenterology and the Italian Association for the Study of the Liver. 2018; 50: 768-79.

11. Xu BB, Lu J, Zheng ZF, Xie JW, Wang JB, Lin JX, et al. The predictive value of the preoperative $\mathrm{C}$-reactive protein-albumin ratio for early recurrence and chemotherapy benefit in patients with gastric cancer after radical gastrectomy: using randomized phase III trial data. Gastric cancer: official journal of the International Gastric Cancer Association and the Japanese Gastric Cancer Association. 2019; 22: 1016-28

12. Muller HG, Wang JL. Hazard rate estimation under random censoring with varying kernels and bandwidths. Biometrics. 1994; 50: 61-76.

13. Hess KR, Serachitopol DM, Brown BW. Hazard function estimators: a simulation study. Statistics in medicine. 1999; 18: 3075-88.

14. Beauchemin N, Arabzadeh A. Carcinoembryonic antigen-related cell adhesion molecules (CEACAMs) in cancer progression and metastasis. Cancer metastasis reviews. 2013; 32: 643-71.

15. Thommen DS, Schumacher TN. T Cell Dysfunction in Cancer. Cancer cell. 2018; 33: 547-62.

16. Knochelmann HM, Dwyer CJ, Bailey SR, Amaya SM, Elston DM, Mazza-McCrann JM, et al. When worlds collide: Th17 and Treg cells in cancer and autoimmunity. Cellular \& molecular immunology. 2018; 15: 458-69.

17. Lambert AW, Pattabiraman DR, Weinberg RA. Emerging Biological Principles of Metastasis. Cell. 2017; 168: 670-91.

18. Kim JG, Lee SJ, Chae YS, Kang BW, Lee YJ, Oh SY, et al. Association between phosphorylated AMP-activated protein kinase and MAPK3/1 expression and prognosis for patients with gastric cancer. Oncology. 2013; 85: 78-85.

19. Aloe S, D'Alessandro R, Spila A, Ferroni P, Basili S, Palmirotta R, et al. Prognostic value of serum and tumor tissue CA 72-4 content in gastric cancer. The International journal of biological markers. 2003; 18: 21-7.

20. Gaspar MJ, Arribas I, Coca MC, Diez-Alonso M. Prognostic value of carcinoembryonic antigen, CA 19-9 and CA 72-4 in gastric carcinoma. Tumour biology : the journal of the International Society for Oncodevelopmental Biology and Medicine. 2001; 22: 318-22.
21. Ye XT, Guo AJ, Yin PF, Cao XD, Chang JC. Overexpression of NUAK1 is associated with disease-free survival and overall survival in patients with gastric cancer. Medical oncology (Northwood, London, England). 2014; 31: 61.

22. Kim DH, Oh SJ, Oh CA, Choi MG, Noh JH, Sohn TS, et al. The relationships between perioperative CEA, CA 19-9, and CA 72-4 and recurrence in gastric cancer patients after curative radical gastrectomy. Journal of surgical oncology. 2011; 104: 585-91.

23. Cetin B, Atalay C, Aslan S, Babacan B, Hatipoglu C, Akinci M, et al. Peritoneal carcinoembryonic antigen level for predicting locoregional and distant spread of gastric cancer. Surgery today. 2005; 35: 919-24.

24. Nakanishi K, Kanda M. The levels of SYT13 and CEA mRNAs in peritoneal lavages predict the peritoneal recurrence of gastric cancer. Gastric Cancer. 2019; 22:1143-52.

25. Ikeda $\mathrm{Y}$, Mori $\mathrm{M}$, Adachi $\mathrm{Y}$, Matsushima $\mathrm{T}$, Sugimachi $\mathrm{K}$, Saku M. Carcinoembryonic antigen (CEA) in stage IV gastric cancer as a risk factor for liver metastasis: a univariate and multivariate analysis. Journal of surgical oncology. 1993; 53: 235-8.

26. Ikeda Y, Oomori H, Koyanagi N, Mori M, Kamakura T, Minagawa S, et al. Prognostic value of combination assays for CEA and CA 19-9 in gastric cancer. Oncology. 1995; 52: 483-6.

27. Xiao J, Ye ZS, Wei SH, Zeng Y, Lin ZM, Wang Y, et al. Prognostic significance of pretreatment serum carcinoembryonic antigen levels in gastric cancer with pathological lymph node-negative: A large sample single-center retrospective study. World journal of gastroenterology. 2017; 23: 8562-9.

28. Li Y, Cao H, Jiao Z, Pakala SB, Sirigiri DN, Li W, et al. Carcinoembryonic antigen interacts with TGF-\{beta\} receptor and inhibits TGF-\{beta\} signaling in colorectal cancers. Cancer research. 2010; 70: 8159-68.

29. Lee YJ, Han SR, Kim NY, Lee SH, Jeong JS, Lee SW. An RNA aptamer that binds carcinoembryonic antigen inhibits hepatic metastasis of colon cancer cells in mice. Gastroenterology. 2012; 143: 155-65.e8.

30. Chang JS, Kim KH, Yoon HI, Hyung WJ, Rha SY, Kim HS, et al. Locoregional relapse after gastrectomy with D2 lymphadenectomy for gastric cancer. The British journal of surgery. 2017; 104: 877-84.

31. Barton MK. False elevations of carcinoembryonic antigen levels are common in patients under surveillance for colorectal cancer recurrence. CA: a cancer journal for clinicians. 2014; 64: 365-6.

32. Litvak A, Cercek A, Segal N, Reidy-Lagunes D, Stadler ZK, Yaeger RD, et al. False-positive elevations of carcinoembryonic antigen in patients with a history of resected colorectal cancer. Journal of the National Comprehensive Cancer Network : JNCCN. 2014; 12: 907-13. 